\title{
Prevalence of thyroid dysfunction and autoimmunity among pregnant women with gestational diabetes mellitus in a tertiary care hospital, Visakhapatnam
}

\author{
Srividya.Nemani ${ }^{1}$, Vamsi Krishna. Kurumeti ${ }^{2 *}$
}

${ }^{1}$ Department of Obstetrics and Gynecology NRIIMS, Sangivalasa, Visakhapatnam, Andhra Pradesh, India
${ }^{2}$ Department of Endocrinology, Seven Hills Hospital, Ram Nagar, Visakhapatnam, Andhra Pradesh, India

Received: 08 September 2020

Accepted: 08 October 2020

\section{*Correspondence:}

Dr. Vamsi Krishna.Kurumeti,

E-mail: dr_vamsi83@yahoo.com

Copyright: ( $)$ the author(s), publisher and licensee Medip Academy. This is an open-access article distributed under the terms of the Creative Commons Attribution Non-Commercial License, which permits unrestricted non-commercial use, distribution, and reproduction in any medium, provided the original work is properly cited.

\begin{abstract}
Background: The most common endocrine conditions encountered in pregnancy are gestational diabetes mellitus (GDM) and thyroid disease which have been known to mutually exist and impact each other to variable extents.

Methods: A Cross sectional study was conducted from September 2019 to February 2020 among 150 pregnant women (75 women with GDM and 75 women without GDM) with $24-28$ weeks of gestation in a tertiary care hospital. Thyroid function tests (Serum TSH, FT4 and TPO antibodies) were evaluated in all the pregnant women. All the information regarding family history of diabetes mellitus, GDM and thyroid disease was determined in both groups and analyzed by statistical methods in SPSS trial version 21.

Results: Obtained data showed that TSH level $(\mathrm{p}=0.14)$ and anti- TPO $(\mathrm{p}=0.04)$ in women with GDM were more than in euglycemic pregnant women and FT4 $(\mathrm{p} \leq 0.001)$ level was lower. There is higher prevalence of hypothyroidism among GDM patients compared to that of euglycaemic women (29.3\% vs $14.6 \%$ ). TPO Ab was positive among $29.3 \%$ of GDM and $8 \%$ of the normal pregnant women $(\mathrm{p}<0.05)$. High prevalence of hypothyroidism was noticed among TPO Ab positive patients in both the groups, but more in the GDM group comparatively indicating that TPO Ab positivity is associated with a higher risk of hypothyroidism in GDM $(\mathrm{p}<0.05)$.

Conclusions: The high prevalence of thyroid disorders and autoimmunity among GDM group warrants the importance of evaluating thyroid function in pregnant women with impaired glucose tolerance, in view of their increased risk of hypothyroidism.
\end{abstract}

Keywords: Autoimmunity, Gestational diabetes mellitus, Pregnancy, Thyroid dysfunction, Visakhapatnam

\section{INTRODUCTION}

Pregnancy is the time of complicated hormonal changes. The two most common endocrine conditions encountered in pregnancy are gestational diabetes mellitus (GDM) and thyroid disease which have been known to mutually exist and impact each other to variable extents. The prevalence of GDM varies as high as $17.5 \%$ as per the reports of International Association of Diabetes in Pregnancy Study Groups (IADPSG). ${ }^{1}$ While the problem of thyroid dysfunction is prevalent in $10-15 \%$ women during the first half of pregnancy which may be hypothyroidism or hyperthyroidism. ${ }^{2}$ Pregnancy has a considerable effect on the thyroid gland and its functioning. ${ }^{3-4}$

Few studies have shown that there is a correlation between thyroid disease and GDM, stating that there is high incidence of hypothyroxinaemia and high Anti TPO titre among patients with GDM, while some proposed that hypothyroid patients are at higher risk of GDM. ${ }^{6-10}$ Adding to this, gestational diabetes mellitus (GDM) and functional abnormalities in the thyroid can have a variety 
of adverse effects on pregnancy outcomes as well as detrimental effects upon foetal neurocognitive development. ${ }^{11,12}$ Overt and subclinical hypothyroidism are the two most common disorders that are associated with adverse outcomes, and together, they are believed to represent a range of autoimmune thyroiditis.

This is often preceded by the detection of serum autoantibodies directed against thyroid cell constituents, of which thyroid peroxidase antibodies (TPO Ab) are considered as marker for the presence of autoimmune thyroid disease, they are found to be positive in $5-10 \%$ of women, during early pregnancy which results in decreased thyroid functional reserve and leads to development of hypothyroidism. ${ }^{13,14}$

There is little data available on the prevalence of thyroid disorders and autoimmunity among pregnant women affected by gestational diabetes. We therefore investigated and compared gestational diabetic patients and euglycaemic pregnant women to assess the prevalence of thyroid dysfunction and autoimmunity and determine its association in pregnant women with GDM.

\section{METHODS}

A hospital based, cross-sectional descriptive study from September 2019 to February 2020 was conducted to estimate the prevalence of thyroid dysfunction and autoimmunity among pregnant women with gestational diabetes mellitus.

For the purpose of the current study, a convenient sample of one hundred and fifty pregnant women (75 women with GDM and 75 women without GDM) in the second trimester with 24-28 weeks of gestation were recruited for the study. The study participants were selected consecutively from the patients attending Obstetrics \& Gynaecology outpatient department irrespective of their gravida status after fulfilling the eligibility criteria in a tertiary care hospital.

\section{Inclusion criteria}

\section{Cases}

Pregnant women with 24-28 weeks of gestation with GDM with age $\geq 18$ years having singleton pregnancies who gave consent to participate in the study.

\section{Controls}

Pregnant women with a singleton pregnancy and a normal 75g OGTT.

\section{Exclusion criteria}

Overt diabetes, history of thyroid dysfunction or prior thyroid surgery, previous head and neck irradiation, history of autoimmune disease, those on medication, which are known to interfere with thyroid function, those on medication, which are known to interfere with immune function, recurrent miscarriages, infertility, age $<18$ years, subjects who are not willing to participate in the study, twin gestation were excluded from the study.

A self-designed, pretested semi structured questionnaire was used to collect the information. A detailed clinical evaluation with emphasis on the symptoms and signs of hypothyroidism, family history of diabetes, obstetric history and clinical examination is done along with anthropometric measurements of height and weight.

Gestational age (GA) was based on their last menstrual period (LMP), with sonography performed if there were discrepancies between fundal height and LMP or if the LMP was uncertain. Diagnosis and exclusion of GDM was done using Oral glucose tolerance test (OGTT) as suggested by HAPO. ${ }^{15} 75$ grams of anhydrous glucose was given after 8 hours of overnight fast and blood samples were drawn in the fasting state, $1 \mathrm{hr}$ after glucose and $2 \mathrm{hrs}$ after oral glucose for plasma glucose estimation. A diagnosis of GDM was made when any one of the following plasma glucose values were exceeded: Fasting plasma glucose $(\mathrm{FPG}) \geq 92 \mathrm{mg} / \mathrm{dL} ; 1 \mathrm{hr}$ Postprandial Plasma Glucose (PPPG) $\geq 180 \mathrm{mg} / \mathrm{dL}$; 2hr Postprandial Plasma Glucose $(\mathrm{PPPG}) \geq 153 \mathrm{mg} / \mathrm{dL}$.

And five $\mathrm{ml}$ of venous blood sample was also withdrawn from the cubital vein of antenatal women recruited into the study for the analysis of TSH, FT4 and TPO antibodies.

Reference ranges suggested by American Thyroid Association (ATA), 2014 were used for TSH interpretation in the present study. ATA recommends the following trimester-specific reference ranges as normal for TSH: First trimester $\quad-\quad 0.1-2.5 \mu \mathrm{IU} / \mathrm{ml}$; Second trimester - 0.2-3.0 $\mu \mathrm{IU} / \mathrm{ml}$; Third trimester - 0.3-3.0 $\mu \mathrm{IU} / \mathrm{ml}$

Subclinical hypothyroidism ( $\mathrm{SCH})$ was defined as per latest ATA guidelines, 2014 as TSH between 3 and $10 \mathrm{mIU} / \mathrm{L}$ with normal serum FT4 levels. Overt Hypothyroidism $(\mathrm{OH})$ was defined as an elevated TSH (>3 mIU/L) in conjunction with a decreased FT4 or a TSH value of $10 \mathrm{mIU} / \mathrm{L}$ or above irrespective of the FT4 level. A diagnosis of clinical hyperthyroidism was done in the presence of supressed or undetectable serum TSH and an elevated FT4.Thyroid peroxidase (TPO) antibodies were considered positive if the titre was more than the upper limit of normal of the assay used i.e, $34 \mathrm{IU} / \mathrm{ml}$.

TSH, FT4 and TPO antibodies were evaluated using chemiluminescent method (Rosche, ELECSYS 2010, Hitachi high technology corporation, Tokyo, Japan).

Cases of GDM were started on medical nutrition therapy and insulin as necessary to maintain the glycemic targets 
as suggested in the Endocrine Society Practice Guidelines of $\mathrm{FPG} \leq 95 \mathrm{mg} / \mathrm{dL}, 1 \mathrm{hr} P P P G \leq 140 \mathrm{mg} / \mathrm{dL}$ and $2 \mathrm{hr}$ $\mathrm{PPPG} \leq 120 \mathrm{mg} / \mathrm{dL}$

SCH was diagnosed based on ATA guidelines and oral LT4 therapy was initiated. Aim of therapy was to maintain TSH in the trimester specific reference range i.e. less $3.0 \mu \mathrm{IU} / \mathrm{ml}$ during the second and third trimesters. Once treatment was initiated or changed, TSH was repeated after 4 weeks. Once TSH became normal, it was repeated every 4-6 weeks until delivery.

\section{Data analysis}

Data was entered in Microsoft excel worksheet 2013 and analysed using SPSS trial version 21. Categorical variables were represented as proportions/percentages and quantitative variables were represented as mean \pm standard deviation.

Independent student $t$ test has been used to determine the significant difference between the means of two groups. Chi- square test is applied wherever applicable to find out significant association. $\mathrm{P}$ value of less than 0.05 was considered as statistical significance at $95 \%$ confidence intervals.

\section{RESULTS}

A total of 150 study participants were included in the study .One group of 75 pregnant women with GDM and another 75 euglycemic pregnant women were studied to compare the prevalence of thyroid dysfunction and autoimmunity in pregnant women with gestational diabetes. The age of the study participants ranged between 18 to 37 years, while mean age of the study participants with GDM is calculated to be $27 \pm 4.12$ and those without GDM is $22.1 \pm 2.52$ years. Regarding baseline characteristics as shown in table 1, GDM women as compared to the euglycemic women were older, more parous and had significant past history of GDM, pregnancy loss and family history of diabetes.

Table 1: Baseline characteristics of the study participants.

\begin{tabular}{|llll|}
\hline Variable & $\begin{array}{l}\text { GDM } \\
\text { women } \\
\text { N }(\%)\end{array}$ & $\begin{array}{l}\text { Non-GDM } \\
\text { women } \\
\text { N }(\%)\end{array}$ & P value \\
\hline Gravida & & & \\
\hline Primigravida & $10(13.3)$ & $35(46.6)$ & $<0.005^{*}$ \\
\hline Multigravida & $65(86.7)$ & $40(53.4)$ & \\
\hline H/o GDM & $10(13.3)$ & 0 & $0.01^{*}$ \\
\hline $\begin{array}{l}\text { H/o pregnancy } \\
\text { loss }\end{array}$ & $12(16)$ & $2(2.6)$ & $<0.0001^{*}$ \\
\hline $\begin{array}{l}\text { Family H/o } \\
\text { DM }\end{array}$ & $20(26.7)$ & $1(1.3)$ & $0.00014^{*}$ \\
\hline
\end{tabular}

*statistically significant
The biochemical characteristics as depicted in Table 2 shows that though the mean serum level of TSH was higher in patients with GDM than non GDM group, however it was not statistically significant. However the mean FT4 was lower and mean Anti TPO antibodies were higher in the pregnant women with GDM, this difference was found to be statistically significant with a $\mathrm{p}$ value of less than 0.05 .

Table 2: Biochemical characteristics of the study participants.

\begin{tabular}{|llll|}
\hline Parameter & $\begin{array}{l}\text { GDM } \\
\text { women } \\
(\text { mean } \pm \text { SD) }\end{array}$ & $\begin{array}{l}\text { Non- GDM } \\
\text { Women } \\
(\text { mean } \pm \text { SD) }\end{array}$ & $\begin{array}{l}\text { P } \\
\text { value }\end{array}$ \\
\hline FPG (mg/dL) & $104.2 \pm 8.1$ & $85.7 \pm 6.1$ & $\begin{array}{l}0.0001 \\
*\end{array}$ \\
\hline $\begin{array}{l}\text { 1h PPPG } \\
\text { (mg/dL) }\end{array}$ & $186.2 \pm 9.3$ & $149.7 \pm 9.7$ & $0.0001^{*}$ \\
\hline $\begin{array}{l}\text { 2h PPPG } \\
\text { (mg/dL) }\end{array}$ & $156.2 \pm 6.2$ & $108.6 \pm 6.7$ & $0.0001^{*}$ \\
\hline TSH ( $\boldsymbol{\mu I U / m l )}$ & $2.66 \pm 1.351$ & $2.18 \pm 1.11$ & 0.14 \\
\hline FT4 (ng/ml) & $0.88 \pm 0.11$ & $1.04 \pm 0.15$ & $<0.0001$ \\
\hline $\begin{array}{l}\text { Anti TPO } \\
\text { (IU/ml) }\end{array}$ & $38.19 \pm 69.79$ & $17.71 \pm 22.13$ & $0.04^{*}$ \\
\hline
\end{tabular}

*statistically significant

On analysis of thyroid dysfunction, among study participants with GDM, $8 \%$ had Subclinical hypothyroidism $(\mathrm{SCH}), 17.3 \%$ had overt hypothyroidism $(\mathrm{OH})$, while among non-GDM patients, 12\% had subclinical hypothyroidism $(\mathrm{SCH})$ and $2.6 \%$ had overt hypothyroidism $(\mathrm{OH})$. No cases of hyperthyroidism was found in either group, though goitre was seen in $46.6 \%$ and $11.6 \%$ of the GDM and non-GDM patients respectively (Figure 1).

Thyroid peroxidase (TPO) antibodies was measured in our study as the marker of thyroid autoimmunity, as depicted in Figure 2, these were positive in $22(29.3 \%)$ pregnant women with GDM and $6(8 \%)$ pregnant women without GDM $(\mathrm{P}<0.05)$.

Table 3: Mean TSH and FT4 values with respect to TPO antibody in both the groups.

\begin{tabular}{|llll|}
\hline Variables & \multicolumn{1}{|c|}{$\begin{array}{c}\text { TPO Ab } \\
\text { positive }\end{array}$} & $\begin{array}{c}\text { TPO Ab } \\
\text { negative }\end{array}$ & P value \\
\hline \multicolumn{4}{|l|}{ GDM patients } \\
\hline TSH & $3.69 \pm 1.40$ & $2.23 \pm 1.08$ & $<0.001^{*}$ \\
\hline FT4 & $0.85 \pm 0.12$ & $0.90 \pm 0.11$ & $0.0087^{*}$ \\
\hline Non-GDM patients & & \\
\hline TSH & $2.65 \pm 1.2$ & $2.13 \pm 1.1$ & 0.27 \\
\hline FT4 & $1.002 \pm 0.01$ & $1.04 \pm 0.15$ & 0.54 \\
\hline
\end{tabular}

*statistically significant

On comparing the mean TSH and FT4 in TPO Ab positive and negative subjects, it was found that the mean TSH was significantly higher and FT4 was significantly 
lower among the TPO Ab positive individuals compared to TPO Ab negative cases in the GDM as well as NonGDM patients, but were not statistically significant in the Non-GDM patients. The mean TSH was also noted to be higher among TPO Ab positive cases of GDM when compared to TPO Ab positive Non-GDM patients (Table 2 ). As regard to hypothyroidism, it was found among more than half of the TPO Ab positive cases $(\mathrm{OH}-40.1 \%$ and $\mathrm{SCH}-13.63 \%$ ), whereas it is only $18.86 \%$ in TPO $\mathrm{Ab}$ negative GDM subjects (Figure 3).

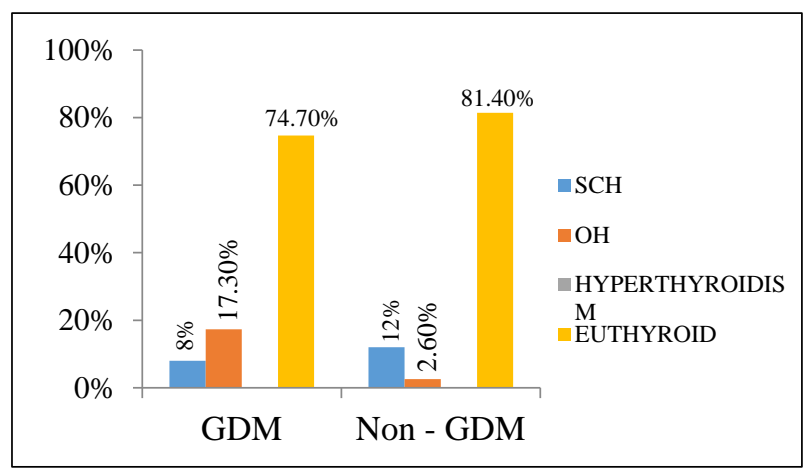

Figure 1: Thyroid dysfunction among the study participants.

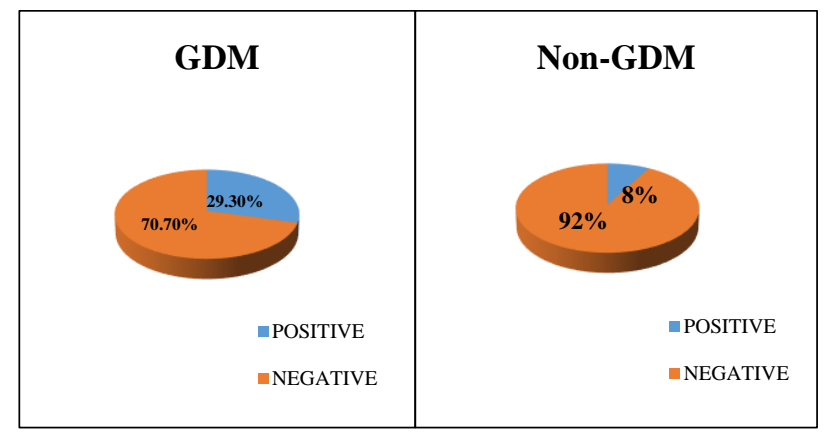

Figure 2: TPO Ab status among the study participants.

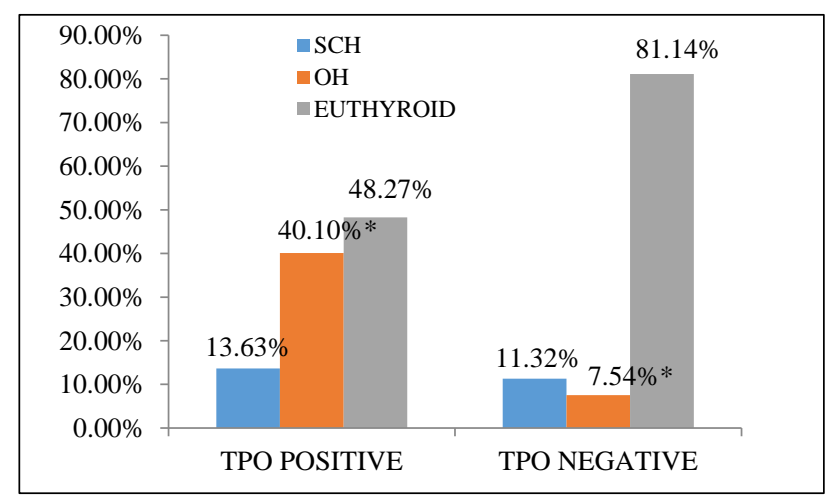

Figure 3: Thyroid dysfunction with respect to TPO positivity in GDM subjects.

Similarly high prevalence of hypothyroidism was noticed among TPO Ab positive Non-GDM subjects (50\% vs $11 \%$. Figure 4 ), indicating that TPO $\mathrm{Ab}$ positivity is associated with a higher risk of hypothyroidism in GDM, and this difference is found to be statistically significant with a $\mathrm{p}$ value of $<0.05$.

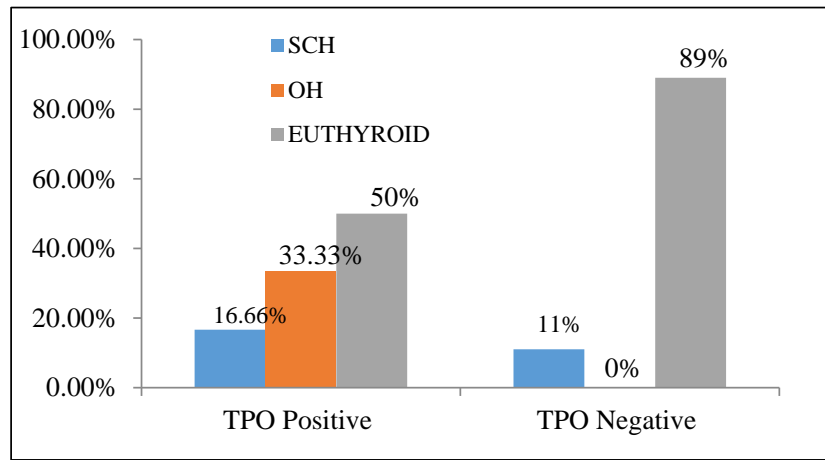

Figure 4: Thyroid dysfunction with respect to TPO positivity in Non-GDM subjects.

\section{DISCUSSION}

Pregnancy leads to complex metabolic changes due to increasing demands of the growing foetus, which affect the endocrine function. The interaction of pregnancy and diabetes mellitus or thyroid disease requires special consideration because of the increased risks to the wellbeing of the mother and the foetus. ${ }^{16}$

In the present study, pregnant women with GDM are compared with Euglycaemic pregnant women to assess the prevalence and association of thyroid dysfunction and autoimmunity in pregnant women with GDM. The patients with GDM were significantly older, more parous and had significant past history of GDM, pregnancy loss and family history of diabetes than their counter group with a $\mathrm{P}$ value of less than 0.001 . This is quite similar to the study findings of Yang $\mathrm{S}$ et al who reported that the incidence of GDM in pregnant women tended to increase with age and those who had a family history of diabetes had a much higher incidence of GDM than those without a family history of diabetes. ${ }^{17}$

The mean FT4 levels in GDM subjects were significantly lower than the normal pregnant women, but the mean TSH values were reported to be higher in GDM patients, though statistically insignificant. The results were comparable to that of the Iranian study by Parham M et al which found a statistically higher TSH levels in GDM subjects compared to the euglycaemic subjects and a lower FT4 levels, though statistically not significant. ${ }^{18}$ Another similar study from Macedonia by Nakova et al found lower FT4 levels $(11.7 \pm 2.4$ vs $13.8 \pm 2.3 \mathrm{pmol} / \mathrm{L}$, $\mathrm{p}<0.05)$ in GDM subjects. ${ }^{8}$ These results are in contrast to the studies by Agarwal et al from UAE 19 and Luisa Ruas et al 20 who showed similar values of FT4 and TSH in GDM and normal pregnancies. This discrepancy might be explained by the high prevalence of iodine deficiency in the baseline population from which our study subjects were selected. 
With regard to the prevalence of thyroid dysfunction, our results showed that there is higher prevalence of hypothyroidism among GDM patients compared to that of euglycaemic women $(25.3 \% \mathrm{Vs} 14.6 \%)$. In the GDM subjects, out of $25.3 \%$ prevalence of hypothyroidism, $8 \%$ had $\mathrm{SCH}$ and $17.3 \%$ had $\mathrm{OH}$. While in the non-GDM subjects, among $14.6 \%$ patients with hypothyroidism, $12 \%$ had $\mathrm{SCH}$ and $2.6 \%$ had $\mathrm{OH}$. Overt hypothyroidism was found to be significantly higher in GDM subjects, while in Non-GDM patients, subclinical hypothyroidism was more common.

This results are consistent with the findings of studies conducted by Fatima et al, Nakova et al and Karakosta et al who stated that hypothyroxinemia is evidently more common among patients with GDM when compared to normal pregnancies and hypothyroid patients are at higher risk of developing GDM which could be due to the shared auto-immunity. ${ }^{6,8,10}$ No cases of hyperthyroidism were found neither in the GDM nor in the Non-GDM group study subjects. Similar findings were reported by Nakova et al. ${ }^{8}$

In contrary to this, the study findings of Iranparvar et al reported hyperthyroidism among 7.1\% of GDM subjects and $1.4 \%$ of euglycemic subjects. ${ }^{21}$ TPO Ab was measured in our study as the marker of thyroid autoimmunity. TPO Ab was positive among $29.3 \%$ of GDM and $8 \%$ of the normal pregnant women $(\mathrm{p}<0.05)$.

The mean TPO Ab titre was also found to be significantly higher in the GDM patients. (38.19 \pm 69.79 vs $17.71 \pm 22.13, \mathrm{p}=0.04)$. In a study by Vitacolonna et al reported the prevalence of TPO Ab positivity as $17.6 \%$ among GDM and an even higher TPO Ab positivity of $26.2 \%$ in postpartum women with history of GDM, possibly establishing the relation between GDM and thyroid autoimmunity. ${ }^{22}$

Though, Nakova et al also found higher TPO Ab positivity among GDM subjects (15\% vs $10 \%)$, it was statistically insignificant. ${ }^{8}$ The prevalence of $8 \%$ thyroid autoimmunity among normal pregnant women is in par with the reports of Ghanavati et al and Mandel et al. ${ }^{13,14}$

The factors predicting TPO Ab positivity in the current study could be older age, multiparity, family history of diabetes and thyroid disease in the first degree relatives. This also could be associated with a higher degree of previous miscarriages in the GDM subjects. Another related study from Italy by Olievieri et al similarly reported the increased risk of thyroid autoimmunity in GDM pregnancies with family history of diabetes and thyroid dysfunction. $^{23}$

To establish the association between thyroid autoimmunity and thyroid dysfunction, the mean FT4 and TSH were compared between TPO Ab positive and TPO Ab negative subjects. Mean TSH was found to be higher and mean FT4 was low among TPO Ab positive subjects when compared to TPO Ab negative subjects in both the groups, although only in GDM patients, this difference was statistically significant. Hypothyroidism was found among more than half of the TPO Ab positive GDM patients compared to less than one-fifth in the TPO Ab negative GDM patients.

Similar high prevalence of hypothyroidism was seen among TPO Ab positive Non-GDM patients (50\% vs $11 \%$ ). The results are consistent with those from Nakova et al who also showed a higher TSH among TPO Ab positive individuals. ${ }^{8}$ Very few studies have studied for this association in GDM patients. Agarwal et al from UAE demonstrated a higher TSH and lower FT4 in TPO positive subjects but failed to show an association between GDM and thyroid dysfunction. ${ }^{19}$ Ruas et al also found no correlation between TPO and thyroid dysfunction. $^{20}$

\section{CONCLUSION}

On the basis of our data, it can be speculated that an increase in thyroid autoimmunity occurs in GDM and that phenomenon is relevant enough to cause thyroid dysfunction either clinical or subclinical. Further studies with repeated measurements of specific biomarkers related to autoimmunity (glutamic acid decarboxylase, islet cell, or insulin antibodies) during pregnancy could investigate whether the association between high TSH and thyroid autoimmunity with gestational diabetes is the consequence of a widespread autoimmune disorder. Keeping in mind the adverse outcomes of maternal hypothyroidism, it is obvious to screen for thyroid dysfunction in pregnancy. The undiagnosed thyroid dysfunction and autoimmunity might potentially further compromise the known maternal and fetal complications associated with GDM. This is one of the few studies to demonstrate the association between thyroid autoimmunity and thyroid dysfunction in GDM women.

\section{Limitations}

As the sample size is very less, the results have to be confirmed in larger cohort studies, also including the analysis of the pregnancy outcomes. The diagnosis of thyroid autoimmunity was based only on detection of TPO Ab. Anti Tg Ab was not utilised in this study due to financial constraints. FT4 estimation was done using chemiluminiscence, which has its own limitations. The FT4 range of the assay was utilised for the diagnosis of hypothyroxinemia due to the lack of population specific reference range in pregnancy.

\section{ACKNOWLEDGMENTS}

Authors would like to thank all the doctors and nurses who facilitated the research process and last but not the least, the study participants who consented and actively participated in the study. 
Funding: No funding sources

Conflict of interest: None declared

Ethical approval: The study was approved by the Institutional Ethics Committee

\section{REFERENCES}

1. ADA. Standards of medical care in diabetes. Diabetes Care. 2014;37:14-80.

2. Bloomgarden ZT. Abnormal thyroid function during pregnancy.2003. Available at http://www. medscape.com /view article/458428. Accessed on 4 August 2020.

3. Yalamanchi S, Cooper DS. Thyroid disorders in pregnancy. Curr Opin Obstet Gynecol. 2015;27(6):406-15.

4. Beneventi F, Locatelli E, Caporali R. Connective tissue diseases and autoimmune thyroid disorders in the first trimester of pregnancy. J Reprod Immunol. 2016;114:32-7.

5. Alexander EK, Pearce EN, Brent GA, et al. Guidelines of the American Thyroid Association for the diagnosis and management of thyroid disease during pregnancy and the postpartum. Thyroid. 2017;27(3):315-89.

6. Fatima SS, Rehman R, Butt Z. Screening of subclinical hypothyroidism during gestational diabetes in Pakistani population. J Matern Fetal Neonatal Med. 2016;29:2166-70.

7. Maleki N, Tavosi Z. Evaluation of thyroid dysfunction and autoimmunity in gestational diabetes mellitus and its relationship with postpartum thyroiditis. Diabet Med. 2015;32:206-12.

8. Nakova V, Krstevska B, Dimitrovski C, Simeonova S, Hadzi-Lega M, Serafimoski V. Prevalence of thyroid dysfunction and autoimmunity in pregnant women with gestational diabetes and diabetes type 1 . Prilozi. 2010;31:51-9.

9. Tudela CM, Casey BM, McIntire DD, Cunningham FG. Relationship of subclinical thyroid disease to the incidence of gestational diabetes. Obstet Gynecol. 2012;119:983-8.

10. Karakosta P, Alegakis D, Georgiou V, Roumeliotaki $\mathrm{T}$, Fthenou E, Vassilaki M, et al. Thyroid dysfunction and autoantibodies in early pregnancy are associated with increased risk of gestational diabetes and adverse birth outcomes. J Clin Endocrinol Metab. 2012;97(12):4464-72.

11. Sepe SJ, Connell FA, Geiss LS, Teutsch SM. Gestational diabetes: incidence, maternal characteristics and perinatal outcome. Diabetes. 1985;34:13-6.

12. Allan WC, Haddow JE, Palomaki GE, Williams JR, Mitchell ML, Hermos RJ, et al. Maternal thyroid deficiency and pregnancy complications: implications for population screening. J Med Screen. 2000;7(3):127-30.

13. Ghanavati A, Casey M, Brian M, Catherine Y, McIntire, Donald D, et al. Pregnancy outcomes in women with thyroid peroxidase antibodies. Obst Gynecol. 2010;116(2):381-6.

14. Mandel SJ. Hypothyroidism and chronic autoimmune thyroiditis in the pregnant state: maternal aspects. Best Pract Res Clin Endocrinol Metab. 2004;18:213-24.

15. HAPO Study Cooperative Research Group. Hyperglycemia and adverse pregnancy outcomes. N Engl J Med. 2008;358:1991-2002.

16. Santini F, Chiovato L, Rocchi R, Marino M, Pinchera A. Influences of thyroid disease in diabetic pregnant women. Ann Super Sanita. 1997;33(3):4415.

17. Yang S, Shi FT, Leung PC, Huang HF, Fan J. Low thyroid hormone in early pregnancy Is associated with an increased risk of gestational diabetes mellitus. J Clin Endocrinol Metab. 2016;101(11):4237-43.

18. Parham M, Asgarani F, Bagherzadeh M, Ebrahimi G, Vafaeimanesh J. Thyroid function in pregnant women with gestational diabetes: Is screening necessary? Thyroid Res Pract. 2015;12:3-7.

19. Agarwal MM, Dhatt GS, Punnose J, Bishawi B, Zayed R. Thyroid function abnormalities and antithyroid antibody prevalence in pregnant women at high risk for gestational diabetes mellitus. Gynecol Endocrinol. 2006;22:261-6.

20. Ruas L, Paiva S, Campos M, Marta E, Lobo A, Cravo A, et al. Prevalence of thyroid antibodies in gestational diabetes mellitus. Endocrine Abstracts. 2007; 14:P338.

21. Iranparvar M, Ghorbani H, Azami A, Darvishkhah H. Thyroid dysfunction and autoimmunity in pregnant women with gestational diabetes mellitus: a cross-sectional study. Int J Adv Med. 2015;2:350-3.

22. Vitacolonna E, Lapolla A, Di Nenno B, Passante A, Bucci I, Giuliani C, et al. Gestational diabetes and thyroid autoimmunity. Int $\mathrm{J}$ Endocrinol. 2012;2012:867415.

23. Olivieri A, Valensise H, Magnani F, Medda E, Angelis SD, D'Archivio M, et al. High frequency of antithyroid autoantibodies in pregnant women at increased risk of gestational diabetes mellitus. European J Endocrinol. 2000;143:741-7.

Cite this article as: Nemani S, Kurumeti VK. Prevalence of thyroid dysfunction and autoimmunity among pregnant women with gestational diabetes mellitus in a tertiary care hospital, Visakhapatnam. Int J Reprod Contracept Obstet Gynecol 2020;9:4621-6. 